\title{
Various perspectives of Two Higgs Doublet models and Naturalness criteria.
}

\author{
Ambalika Biswas* \\ S N BOSE NATIONAL CENTRE FOR BASIC SCIENCES \\ E-mail: ani73biswas@gmail.com \\ Amitabha Lahiri \\ S N BOSE NATIONAL CENTRE FOR BASIC SCIENCES \\ E-mail: amitabha@boson.bose.res.in
}

\begin{abstract}
We study the implication of a criterion of naturalness for a broad class of two Higgs doublet models (2HDMs). In particular, we assume the cancellation of quadratic divergences in what are called the type I, type II, lepton-specific and flipped 2HDMs. This results in a set of relations among masses of the physical scalars and coupling constants, a generalization of the Veltman conditions of the Standard Model. With a softly broken U(1) symmetry, we have studied the various limiting values of the scalar mixing angles $\alpha$ and $\beta$. These correspond to the Standard Model Higgs particle being the lighter CP-even scalar (alignment) or the heavier CP-even scalar (reverse alignment), and also the limit in which some of the Yukawa couplings of this particle are of the opposite sign from the vector boson couplings (wrong sign). Imposing further the constraints from the electroweak T-parameter (or $\rho$ parameter), stability and perturbative unitarity conditions produce a range for the masses of each of the remaining physical scalars. We also calculate the $\mathrm{h} \rightarrow \gamma \gamma$ decay rate in the wrong sign limit.
\end{abstract}

The talk is based on the below two papers.

1. "Masses of physical scalars in two Higgs doublet models" by A.Biswas and A.Lahiri published in PHYSICAL REVIEW D 91, 115012 (2015).

2."Alignment, reverse alignment, and wrong sign Yukawa couplings in two Higgs doublet models" by A.Biswas and A.Lahiri published in PHYSICAL REVIEW D 93, 115017 (2016).

38th International Conference on High Energy Physics

3-10 August 2016

Chicago, USA

\footnotetext{
* Speaker.

${ }^{\dagger}$ Dedicated to my husband.
} 


\section{Introduction}

Some unanswered questions, including the origin of neutrino masses, dark matter and CP violation, keep the door open for physics beyond the Standard Model. The simplest extensions of the Standard Model are two Higgs doublet models (2HDMs) (for a recent review see [1]). Among the various motivations for $2 \mathrm{HDMs}$, the one that is important to us, is their use in models of dark matter.

We will work with the scalar potential [2]

$$
\begin{aligned}
V= & \lambda_{1}\left(\left|\Phi_{1}\right|^{2}-\frac{v_{1}^{2}}{2}\right)^{2}+\lambda_{2}\left(\left|\Phi_{2}\right|^{2}-\frac{v_{2}^{2}}{2}\right)^{2}+\lambda_{3}\left(\left|\Phi_{1}\right|^{2}+\left|\Phi_{2}\right|^{2}-\frac{v_{1}^{2}+v_{2}^{2}}{2}\right)^{2} \\
& +\lambda_{4}\left(\left|\Phi_{1}\right|^{2}\left|\Phi_{2}\right|^{2}-\left|\Phi_{1}^{\dagger} \Phi_{2}\right|^{2}\right)+\lambda_{5}\left|\Phi_{1}^{\dagger} \Phi_{2}-\frac{v_{1} v_{2}}{2}\right|^{2}
\end{aligned}
$$

with real $\lambda_{i}$. This potential is invariant under the global U(1) symmetry $\Phi_{1} \rightarrow e^{i \theta} \Phi_{1}, \Phi_{2} \rightarrow \Phi_{2}$, except for a soft breaking term $\lambda_{5} v_{1} v_{2} \Re\left(\Phi_{1}^{\dagger} \Phi_{2}\right)$. This in turn avoids FCNCs.

Physical Higgs bosons are $\xi^{ \pm}, \mathrm{A}, \mathrm{H}$ and h with $\tan \beta=\frac{v_{2}}{v_{1}}, v=\sqrt{v_{1}^{2}+v_{2}^{2}}=246 \mathrm{GeV}$.

\section{Constraints imposed on the 2HDMs}

Cancellation of quadratic divergences in the scalar masses gives rise to mass relations, which we may call the Veltman conditions [3] for the 2HDMs being considered, $2 \operatorname{Tr} G_{e}^{1} G_{e}^{1 \dagger}+6 \operatorname{Tr} G_{u}^{1 \dagger} G_{u}^{1}+6 \operatorname{Tr} G_{d}^{1} G_{d}^{1 \dagger}=\frac{9}{4} g^{2}+\frac{3}{4} g^{2}+6 \lambda_{1}+10 \lambda_{3}+\lambda_{4}+\lambda_{5}$ $2 \operatorname{Tr} G_{e}^{2} G_{e}^{2 \dagger}+6 \operatorname{Tr} G_{u}^{2 \dagger} G_{u}^{2}+6 \operatorname{Tr} G_{d}^{2} G_{d}^{2 \dagger}=\frac{9}{4} g^{2}+\frac{3}{4} g^{2}+6 \lambda_{2}+10 \lambda_{3}+\lambda_{4}+\lambda_{5}$ where $g, g^{\prime}$ are the $S U(2)$ and $U(1)_{Y}$ coupling constants and $G_{e}^{1}$ etc. are complex $3 \times 3$ matrices in generation space containing the Yukawa coupling constants.

The other constraints are the perturbativity condition, $\lambda_{i} \leq 4 \pi[4]$, stability conditions [5] and perturbative unitarity conditions [6].

Further constraints come from the modification of $\rho$ parameter from new Physics corrections, $\rho=\frac{m_{W}^{2}}{\cos \theta_{w}^{2} m_{Z}^{2}}$. Recent bounds on $\delta \rho$ is $\delta \rho=-0.0002 \pm 0.0007$.

\section{Limits of $2 \mathrm{HDMs}$}

\subsection{Alignment Limit}

Here ' $h$ ' is the SM Higgs whose couplings with the fermions and vector bosons are the same as that of the SM Higgs and $\beta-\alpha=\frac{\pi}{2}$. Imposition of all of the above constraints give mass bounds of the physical Higgs bosons. They have been plotted in fig. 1. We find that, $450 \mathrm{GeV} \lesssim$ $m_{H} \lesssim 620 \mathrm{GeV}, 550 \mathrm{GeV} \lesssim m_{\xi} \lesssim 700 \mathrm{GeV}$. The above mass ranges vary between a few $\mathrm{GeV}$ for the various $2 \mathrm{HDMs}$. Direct searches predict $m_{\xi}>100 \mathrm{GeV}$ and our results agree with this lower bound. Moreover the degeneracy in the masses of the physical Higgs bosons for large enough $\tan \beta$ is evident from our plots. 


\subsection{Reverse Alignment Limit}

In this limit ' $\mathrm{H}$ ' is treated as the SM Higgs and $\beta \approx \alpha$. Various constraints on the parameter space gives no mass ranges for the physical Higgs boson masses thus making the Reverse alignment limit inconsistent with Naturalness.

\subsection{Wrong Sign Limit}

Wrong sign limit [7] is considered along with the Alignment limit where $\frac{h \bar{D} D}{h V V}<0$ or $\frac{h \bar{U} U}{h V V}<0$, where $\mathrm{h}$ is the SM-like Higgs.

Now the type-II Higgs-fermion Yukawa couplings normalized w.r.t. SM:

$$
\begin{aligned}
& h \bar{D} D:-\frac{\sin \alpha}{\cos \beta}=-\sin (\beta+\alpha)+\cos (\beta+\alpha) \tan \beta \\
& h \bar{U} U: \frac{\cos \alpha}{\sin \beta}=\sin (\beta+\alpha)+\cos (\beta+\alpha) \cot \beta
\end{aligned}
$$

where if $\sin (\beta+\alpha)=1 \Rightarrow h \bar{D} D=-1$ and $h \bar{U} U=+1$. Thus Wrong Sign and Alignment limit are achieved for $\sin (\beta-\alpha) \sim 1$ and $\sin (\beta+\alpha) \sim 1$.

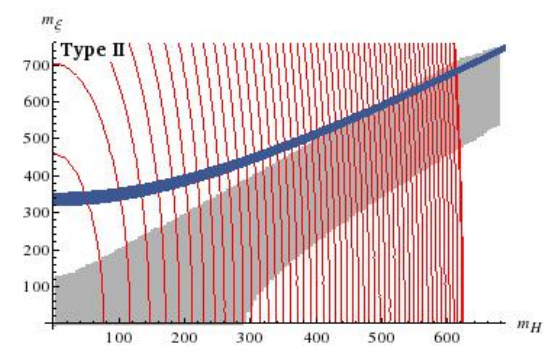

Figure 1: Alignment limit

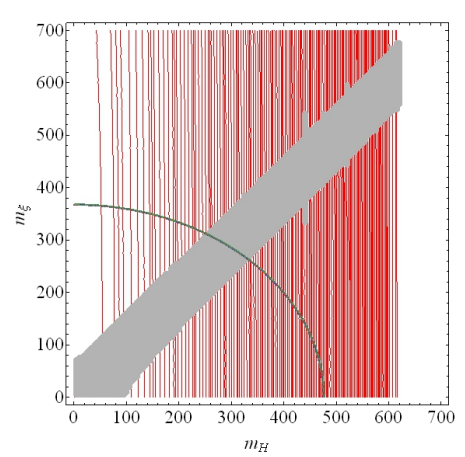

Figure 3: Wrong Sign Limit, $\tan \beta 17$

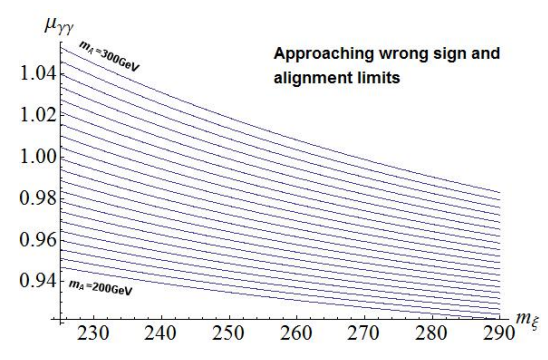

Figure 2: Diphoton decay width

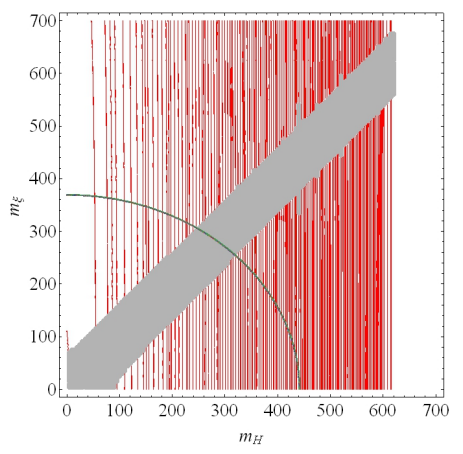

Figure 4: Wrong Sign Limit, $\tan \beta 20$

The wrong sign limit approaches the alignment limit for $\tan \beta \approx 17$ as shown in fig. 3. For $\tan \beta=17,250 \mathrm{GeV} \lesssim m_{H} \lesssim 330 \mathrm{GeV}$ and $260 \mathrm{GeV} \lesssim m_{\xi} \lesssim 310 \mathrm{GeV}$. At higher values of $\tan \beta$, both ranges become narrower and move down on the mass scale (fig. 4). 


\section{Modification of Higgs diphoton decay width}

The Higgs-diphoton decay width is calculated using the formula [8]

$$
\Gamma(h \rightarrow \gamma \gamma)=\frac{G_{\mu} \alpha^{2} m_{h}^{3}}{128 \sqrt{2} \pi^{3}}\left|\sum_{f} N_{c} Q_{f}^{2} g_{h f f} A_{1 / 2}^{h}\left(\tau_{f}\right)+g_{h V V} A_{1}^{h}\left(\tau_{W}\right)+\frac{m_{W}^{2} \lambda_{h \xi^{+} \xi^{-}}}{2 c_{W}^{2} M_{\xi^{ \pm}}^{2}} A_{0}^{h}\left(\tau_{\xi^{ \pm}}\right)\right|^{2} .
$$

where, $g_{h t t}=\frac{\cos \alpha}{\sin \beta}, g_{h b b}=-\frac{\sin \alpha}{\cos \beta}, g_{h W W}=\sin (\beta-\alpha), \lambda_{h \xi^{+} \xi^{-}}=\cos 2 \beta \sin (\beta+\alpha)+2 c_{W}^{2} \sin (\beta-$ $\alpha), c_{W}=\cos \theta_{W}$, with $\theta_{W}$ being the Weinberg angle. The decay rate does not depend on the type of the 2HDM. In the case of the CP even Higgs boson $h$,

$$
\begin{gathered}
A_{1 / 2}^{h}=-2 \tau[1+(1-\tau) f(\tau)], A_{1}^{h}=2+3 \tau+3 \tau(2-\tau) f(\tau), \\
f(\tau)= \begin{cases}\arcsin ^{2} \sqrt{1 / \tau}, & \tau \geq 1 \\
-\frac{1}{4}\left[\log \frac{1+\sqrt{1-\tau}}{1-\sqrt{1-\tau}}-i \pi\right]^{2}, & \tau<1\end{cases}
\end{gathered}
$$

The plot for diphoton decay width is plotted in fig. 2 for two limits of 2HDMs.

The relative diphoton decay width increases as $m_{A}$ increases. Maximum value of about $6 \%$ is achieved as compared to the SM value for the wrong sign limit and thus it throws light on BSM Physics. Though a peak at $750 \mathrm{GeV}$ was observed by ATLAS and CMS, but 2HDMs will not advocate it if Naturalness holds.

\section{References}

[1] G. C. Branco, P. M. Ferreira, L. Lavoura, M. N. Rebelo, M. Sher and J. P. Silva, "Theory and phenomenology of two-Higgs-doublet models," Phys. Rept. 516, 1 (2012)

[2] J. F. Gunion, H. E. Haber, G. L. Kane and S. Dawson, "Errata for the Higgs hunter's guide," arXiv:hep-ph/9302272.

[3] M. J. G. Veltman, "The Infrared - Ultraviolet Connection,” Acta Phys. Polon. B 12, 437 (1981), C. Newton and T. T. Wu, "Mass relations in the two Higgs doublet model from the absence of quadratic divergences," Z. Phys. C 62, 253 (1994).

[4] S. Kanemura, T. Kasai and Y. Okada, "Mass bounds of the lightest CP even Higgs boson in the two Higgs doublet model,” Phys. Lett. B 471, 182 (1999) doi:10.1016/S0370-2693(99)01351-9 [hep-ph/9903289].

J. F. Gunion and H. E. Haber,

[5] M. Sher, "Electroweak Higgs Potentials and Vacuum Stability," Phys. Rept. 179, 273 (1989).

[6] B. W. Lee, C. Quigg and H. B. Thacker, "Weak Interactions at Very High-Energies: The Role of the Higgs Boson Mass,” Phys. Rev. D 16, 1519 (1977).

[7] P. M. Ferreira, J. F. Gunion, H. E. Haber and R. Santos, "Probing wrong-sign Yukawa couplings at the LHC and a future linear collider," Phys. Rev. D 89, no. 11, 115003 (2014) [arXiv:1403.4736 [hep-ph]], P. M. Ferreira, R. Guedes, M. O. P. Sampaio and R. Santos, "Wrong sign and symmetric limits and non-decoupling in 2HDMs," JHEP 1412, 067 (2014), P. M. Ferreira, R. Guedes, J. F. Gunion, H. E. Haber, M. O. P. Sampaio and R. Santos, "The Wrong Sign limit in the 2HDM," arXiv:1410.1926 [hep-ph].

[8] A. Djouadi, "The Anatomy of electro-weak symmetry breaking. II. The Higgs bosons in the minimal supersymmetric model," Phys. Rept. 459, 1 (2008) [hep-ph/0503173]. 